\title{
Novel Technique of Endovascular Retrograde Circulatory Support in Patients with Acute Coronary Syndrome: Emphasis on Myocardium Eunctional State
}

DOI: $10.17691 / \mathrm{stm} 2015.7 .4 .02$

Received June 23, 2015

E.B. Shakhov, MD, PhD, Head of Experimental Laboratory, Department of Oncology, Postgraduate Faculty';

D.V. Volkov, Reanimatologist';

E.B. Petrova, MD, PhD, Associate Professor, Department of Radiodiagnosis, Postgraduate Faculty';

E.S. Timoschenko, Cardiologist, Head of the City Cardiodispensary²;

S.G. Erofeyeva, Cardiologist, Deputy Head of the City Cardiodispensary2;

A.A. Nekrasov, MD, DSc, Professor, Department of Faculty and Polyclinic Therapy

1Nizhny Novgorod State Medical Academy, 10/1 Minin and Pozharsky Square, Nizhny Novgorod, 603005, Russian Federation;

${ }_{2}^{2}$ City Clinical Hospital No.5, 34 Nesterova St., Nizhny Novgorod, 603005, Russian Federation

The aim of the investigation is to evaluate the efficacy of the novel technique of endovascular retrograde circulatory support of the cardiac muscle according to the data obtained by studying the main functional parameters of the left ventricular myocardium in the medium terms after complete high-risk radioendovascular revascularization carried out in patients with acute coronary syndrome (ACS) with ST-elevation and nonST-elevation and multivessel lesion of the coronary arterial bloodstream.

Materials and Methods. The results of treating 24 patients with ACS and initial myocardial dysfunction have been analyzed. Group 1 ( $n=12$ ) included ACS patients with ST-elevation, patients of group $2(n=12)$ were with non-ST-elevation. Both groups were divided into equal subgroups: subgroup A comprised patients with retrograde perfusion circulatory support; whereas subgroup B included patients without retrograde perfusion support.

Results. In group 1 complete myocardium function was observed to normalize in 7 individuals (in 6 and 1 patients in subgroups $A$ and $B$, respectively) 12 months after the operation (medium term). In group 2 complete normalization of the myocardium function occurred in 6 individuals (in 4 and 2 patients in subgroups $A$ and B, respectively) in the same period. Patients in subgroups A had significantly better parameters of local contractility and diastolic function 12 months after the operation compared to the patients from subgroups $B$.

Conclusion. Application of the novel technique of retrograde perfusion of the left ventricular anterior wall during complete endovascular revascularization in high-risk patients with ACS results in essential improvement of the results.

Key words: acute coronary syndrome; retroperfusion; circulatory support; myocardial function.

At present the expedience of circulatory support systems in patients with myocardium perfusion impairment is one of the topics being intensively discussed among interventional cardiologists [1]. On the one hand, European and American guidelines refer systemic methods of circulatory support to the second class of recommendations and prove their efficiency in the correction of cardiodynamics in patients with acute coronary syndrome (ACS) with ST-elevation (STE-ACS) on the electrocardiogram [2-4], on the other hand, recommendations do not strictly deny the possibility of their application in patients with ACS non-ST-elevation (NSTE-ACS) in case of high-risk percutaneous intervention [5-6]. This situation needs further investigations to reveal the effect of various techniques of supported circulation directly on the coronary dynamics, and indirectly on the functional myocardium activity in patients with life-threatening atherothrombotic damage of the coronal arterial bloodstream [7-9]. The development and study of specific applications of systemic and local methods of circulatory support in patients with STE-ACS and NSTE-ACS, and also their effect on the cardiac muscle function in different terms after high-risk percutaneous intervention is one of the most vital branch of modern cardiology, demanding further consideration.

The aim of the investigation is to evaluate the efficacy of the novel technique of endovascular retrograde circulatory support of the cardiac muscle according to the data obtained by studying the main functional parameters of the left ventricular myocardium in the medium terms after full high-risk radioendovascular revascularization performed on patients with acute coronary syndrome with ST-elevation and non-STelevation and multivessel lesion of the coronary arterial bloodstream.

For contacts: Shakhov Evgeniy Borisovich, e-mail: medinn-ngma@yandex.ru 
Materials and Methods. The results of treating 24 patients with ACS at the age of 41-75 years (average age $62.4 \pm 11.8$ years) staying in City Clinical Hospital No.5 for 2 years (from April 2012 till May 2014) have been analyzed. On the basis of the detailed examination of the clinical, electrocardiographic (ECG) and echocardiographic (echoCG) picture at all stages of investigation the patients were divided into two equal groups: patients with STE-ACS comprised group 1, and patients with NSTE-ACS were included into group 2.

The study complies with the Declaration of Helsinki (the Declaration was passed in Helsinki, Finland, June, 1964, and revised in October, 2000, Edinburg, Scotland) and was performed following approval by the Ethic Committee of Nizhny Novgorod State Medical Academy. Written informed consent was obtained from every patient.

During the year before hospitalization for ACS the picture of coronary heart disease in patients of group 1 was presented by stable exertional angina in 8 and progressing angina in 3 individuals. One of the examined patients has not got previously valid coronary history. In patients of group 2 stable exertional angina was present in 7 people and progressing angina in 4 . One person was diagnosed angina for the first time directly during his visit to the cardiologist a month before hospitalization for ACS. In group 1 there were 5 patients with myocardial infarction (MI) in the history: 3 cases with non-Q-wave infarction, and 2 cases with Q-wave infarction. In group 2 there were revealed 4 patients with $\mathrm{MI}$ in the history: non-Q-wave infarction in 2 patients and Q-wave infarction also in 2. During 12 months before hospitalization for ACS none of the examined patients suffer Ml. Concomitant pathology in the form of hypertension, diabetes mellitus type 2 was found in 10 patients of group 1 and in 11 ones in group 2 .

Important criteria for inclusion of the patients in our investigation were: evidence of acute myocardial ischemia on the anterior wall of the left ventricle (LV), high risk of fatal and non-fatal cardiac events, angiographically documented multivessel (complex) atherosclerotic damage of the coronary cardiac bloodstream with localization of the clinical picture-dependent lesion in the zone of the anterior coronary circulation (Table 1).

So, in all analyzed patients symptom-associated lesions (stenosis more than $70 \%$ ) were located in the zone of the left coronary artery trunk (LCAT) or anterior descending artery (ADA) with transition to the large diagonal branches (DB),
Table 1

\section{Characteristics of the patients suffering from acute} coronary syndrome

\begin{tabular}{|lc|}
\hline \multicolumn{1}{|c}{$\begin{array}{c}\text { Clinical features of patients with acute } \\
\text { coronary syndrome }\end{array}$} & $\begin{array}{c}\text { Quantity } \\
\text { of patients } \\
\text { (abs. number/\%) }\end{array}$ \\
\hline Group 1 (STE-ACS) & $12 / 50$ \\
\hline Group 2 (NSTE-ACS) & $12 / 50$ \\
\hline Males & $18 / 75$ \\
\hline Females & $6 / 25$ \\
\hline $\begin{array}{l}\text { Clinical picture-depended lesion: } \\
\text { LCAT }\end{array}$ & $15 / 63$ \\
\hline $\begin{array}{l}\text { ADA } \\
\text { ADA-DB }\end{array}$ & $18 / 75$ \\
\hline $\begin{array}{l}\text { Stratification of hospital lethality risk } \\
\text { and probability of myocardial infarction: } \\
\text { high risk according to the TIMI risk scores }\end{array}$ & $24 / 54$ \\
\hline \multicolumn{2}{l}{ high risk according to the GRACE risk scores } \\
\hline $\begin{array}{l}\text { Stratification of surgical risk and complexity } \\
\text { of percutaneous coronary intervention - } \\
\text { high risk according to the Syntax Score }\end{array}$ & $24 / 100$ \\
\hline
\end{tabular}

N o t e. STE-ACS: acute coronary syndrome with ST-elevation; NSTE-ACS: acute coronary syndrome with non-ST-elevation; LCAT: left coronary artery trunk; ADA: anterior descending artery; DB: diagonal branches.

as revealed by selective coronarography. Concomitant stenosis of the posterior coronary circulation were visualized in the zone of the circumflex artery, right coronary arteries or in their large branches [10-12].

As a part of emergency invasive strategy high-risk patients underwent urgent endovascular correction of the complex multivessel atherosclerotic lesion of the coronary bloodstream (Figure 1) following selective coronarography. Mean "door-balloon" time in our study amounted to $74.8 \pm 11.4 \mathrm{~min}$.
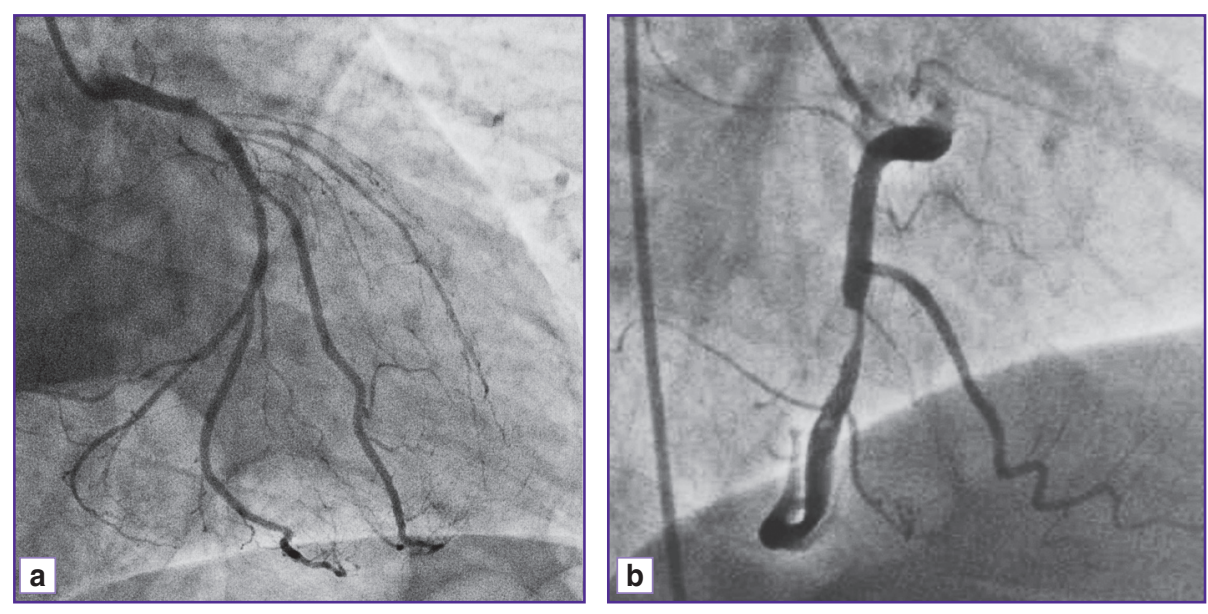

Figure 1. Multivessel complex lesion of the coronary circulation (acute coronary syndrome with ST-elevation): (a) bifurcation trunk of the left coronary artery, anterior descending artery (acute occlusion), circumflex artery; (b) stenosis of the right coronary artery 
Prior to angioplasty and stenting each group of patients with ACS was divided into two subgroups (A and $B$ ) equal in quantity and quality, depending on performing or not local intraoperative circulatory support.

Subgroup A comprised patients, who during highrisk percutaneous coronary intervention underwent retrograde perfusion of the ischemic myocardium in the region of the anterior wall of the LV, subgroup $B$ included patients without supporting blood supply of the cardiac muscle. Reperfusion was carried out using proprietory technique (patent decision No.2014111410/14(08016) of 25.03.2014). The main idea of it is that in the process of endovascular correction of the damaged coronary arteries selective temporary arterialization of the large cardinal vein is performed not synchronically with diastole of the heart. For this purpose, puncture of the subclavian or jugular vein on the right or left was performed with the subsequent placement of the delivery system $8 \mathrm{~F}$ to the coronary sinus orifice. Using the delivery system, standard two-lumen retroperfusion balloon catheter (of Swan-Ganz type) 6F was inserted selectively to the proximal part of the large cardinal vein, draining blood from the anterior wall of the LV. Parallel with selective catheterization of the large cardinal vein the radial artery was punctured from the right and left for collection of arterial autoblood with the following installation of a standard radial introducer $5 \mathrm{~F}$ into the arterial lumen. Prior to retroperfusion the balloon was dilatated till the complete occlusion of the proximal segment of the large cardinal vein through one of the lumens of the retroperfusion balloon catheter, and thereafter a free internal lumen of the retroperfusion balloon catheter was connected to the perfusion unit BP-05 (Avangard, Russia). To withdraw arterial autoblood, perfusion unit was connected to the arterial introducer, placed in the radial artery. The time of retroperfusion corresponded to the time of antegrage blood flow blockage in the process of stent installation (60-70 s). Perfusion rate in the process of blocking antegrade blood flow did not exceed $40 \mathrm{ml} / \mathrm{min}$ in the anterior coronary region. It was not synchronized with the heart diastole, but was carried on continuously till restoration of the adequate antegrade blood flow through LCAT, ADA and DB. This technique of conducting retroperfusion facilitates selective retrograde perfusion of the clinical picture-dependent ischemic zone of myocardium.

To evaluate the efficacy of the endovascular intervention with or without supporting circulation and in medium terms after intervention (after 12 months) all patients underwent ECG and echoCG examination. Ultrasound scanner Vivid 7 Pro (GE Medical Systems, Norway) with 3.0-3.5 MHz transducers, 2D-mode and Doppler imaging was used to perform echocardiography. Main attention was paid to the parameters of LV function: ejection fraction (EF), impaired local contractility (ILC), early-to-late left ventricular filling ratio $(E / A)$.

Statistical data processing was performed by means of the licensed program Statistica 8.0. The results were presented as mean \pm standard deviation $(\mathrm{M} \pm \mathrm{sd})$. Nonparametric statistical analysis of the obtained data by means of Wilcoxon paired signed rank test was used to compare two dependent variables, and Mann-Whitney U-test to compare two independent variables. Comparative analysis of patients' survival in the examined groups was conducted with the help of Kaplan-Meier curves using nonparametric statistical Gehan-Wilcoxon test. All statistical comparisons were considered significant at $p \leqslant 0.05$ [13].

Results. In both groups and subgroups the emphasis was placed on the maximally complete myocardium revascularization with obligatory primary correction of clinical picture-dependent lesion in the zone of anterior coronary circulation. Before the operative intervention all 12 patients in group 1 in both subgroups were found to have abnormal basic echoCG parameters of LV function, initial systolic (SD) and diastolic (DD) dysfunction, as well as ILC (Table 2). DD in both subgroups was of type 1 (impaired relaxation).

In group 2 SD, DD and ILC were revealed in all of the 12 patients before the operative treatment (Table 3). DD in both subgroups was of type 1 (impaired relaxation).

Within the framework of this investigation endovascular intervention was successfully performed in all examined patients of group 1 and 2. Survival in early (within 12 months) and medium (after 12 months) control terms after the operation amounted to $100 \%$. Only 14 months after radiosurgical correction one patient from group 1, subgroup B, died of cardiac rhythm disturbances (Figure 2).

During follow-up period 12 months after complete myocardium revascularization statistically significant

Table 2

Initial basic functional parameters of the left ventricular myocardium in patients of group 1

\begin{tabular}{|lccc}
\hline \multicolumn{1}{c}{ Parameters } & Subgroup A & Subgroup B & $\mathbf{p}$ \\
\hline LV EF $(\%)$ & $42.0 \pm 0.9$ & $42.3 \pm 0.8$ & 0.669 \\
\hline ILC & $1.50 \pm 0.15$ & $1.48 \pm 0.15$ & 0.831 \\
\hline E/A & $0.69 \pm 0.15$ & $0.66 \pm 0.12$ & 0.831 \\
\hline
\end{tabular}

$\mathrm{N}$ o te. LV EF: left ventricular ejection fraction; ILC: impaired local contractility; E/A: indicator of diastolic function.

Table 3

Initial basic functional parameters of the left ventricular myocardium in patients of group 2

\begin{tabular}{lccc}
\hline Parameters & Subgroup A & Subgroup B & $\mathbf{p}$ \\
\hline LV EF (\%) & $45.2 \pm 1.5$ & $44.3 \pm 1.6$ & 0.378 \\
\hline ILC & $1.40 \pm 0.13$ & $1.35 \pm 0.09$ & 0.471 \\
\hline E/A & $0.74 \pm 0.10$ & $0.73 \pm 0.09$ & 0.810 \\
\hline
\end{tabular}

$\mathrm{N}$ o t e. LV EF: left ventricular ejection fraction; ILC: impaired local contractility; E/A: indicator of diastolic function. 
Figure 2. Survival of patients in group 1 and 2

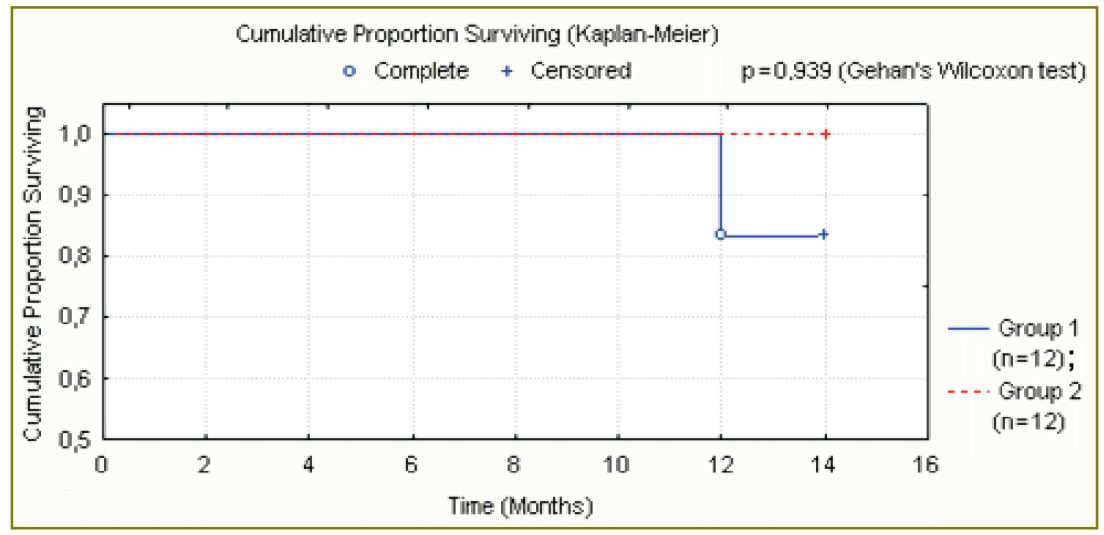

Table 4

Basic functional parameters of the left ventricular myocardium in patients of group 1 a year before and after intervention $(p=0.027)$

\begin{tabular}{|lcc|}
\hline \multicolumn{1}{|c}{ Parameters } & Initially & A year later \\
\hline & Subgroup $\boldsymbol{A}$ & \\
\hline LV EF $(\%)$ & $42.0 \pm 0.9$ & $59.3 \pm 4.8$ \\
\hline ILC & $1.50 \pm 0.15$ & $1.0 \pm 0.0$ \\
\hline E/A & $0.69 \pm 0.15$ & $1.16 \pm 0.04$ \\
\hline & Subgroup B & \\
\hline LV EF $(\%)$ & $42.3 \pm 0.8$ & $56.2 \pm 5.1$ \\
\hline ILC & $1.48 \pm 0.15$ & $1.05 \pm 0.02$ \\
\hline E/A & $0.66 \pm 0.12$ & $1.01 \pm 0.10$ \\
\hline
\end{tabular}

$\mathrm{N}$ o t e. LV EF: left ventricular ejection fraction; ILC: impaired local contractility; E/A: indicator of diastolic function.
Table 5

Basic functional parameters of the left ventricular myocardium in patients of group 2 a year before and after intervention $(p=0.027)$

\begin{tabular}{|lcc|}
\hline \multicolumn{1}{c}{ Parameters } & $\begin{array}{c}\text { Initially } \\
\text { Subgroup } \boldsymbol{A}\end{array}$ & A year later \\
\hline LV EF $(\%)$ & $45.2 \pm 1.5$ & $55.0 \pm 2.8$ \\
\hline ILC & $1.40 \pm 0.13$ & $1.02 \pm 0.03$ \\
\hline E/A & $0.74 \pm 0.10$ & $1.18 \pm 0.06$ \\
\hline & Subgroup $\boldsymbol{B}$ & \\
\hline LV EF $(\%)$ & $44.3 \pm 1.6$ & $51.7 \pm 2.3$ \\
\hline ILC & $1.35 \pm 0.09$ & $1.10 \pm 0.08$ \\
\hline E/A & $0.73 \pm 0.09$ & $1.04 \pm 0.05$ \\
\hline
\end{tabular}

$\mathrm{N}$ o t e. LV EF: left ventricular ejection fraction; ILC: impaired local contractility; E/A: indicator of diastolic function.

Table 6

Basic functional parameters of the left ventricular myocardium in patients with acute coronary syndrome in subgroups a year after intervention

\begin{tabular}{lcccccc}
\hline \multirow{2}{*}{ Parameters } & \multicolumn{2}{c}{ Group 1 } & STE-ACS $(\mathbf{n = 1 2})$ & \multicolumn{3}{c}{ Group 2 } \\
& Subgroup A & Subgroup B & $\mathbf{p}$ & Subgroup A & Subgroup B & $\mathbf{p}$ \\
\hline LV EF $(\%)$ & $59.3 \pm 4.8$ & $56.2 \pm 5.1$ & 0.240 & $55.0 \pm 2.8$ & $51.7 \pm 2.3$ & 0.030 \\
\hline ILC & $1.0 \pm 0.0$ & $1.05 \pm 0.02$ & 0.010 & $1.02 \pm 0.03$ & $1.10 \pm 0.08$ & 0.029 \\
\hline E/A & $1.16 \pm 0.04$ & $1.01 \pm 0.10$ & 0.019 & $1.18 \pm 0.06$ & $1.04 \pm 0.05$ & 0.006 \\
\hline
\end{tabular}

N o t e. STE-ACS: acute coronary syndrome with ST-elevation; NSTE-ACS: acute coronary syndrome with non-ST-elevation; LV EF: left ventricular ejection fraction; ILC: impaired local contractility; E/A: indicator of diastolic function.

improvement of all basic functional myocardium parameters were established in all patients of group 1 (Table 4).

Complete normalization of systolic, diastolic and contractile LV functions was observed in 7 patients (in all 6 patients in subgroup $A$, and only in 1 in subgroup $B$ ). Abnormal basic functional myocardium parameters were revealed in 5 examined individuals from subgroup $B$ : in 3 patients DD (E/A - 0.92 \pm 0.01$)$ and ILC (ILC index -
$1.06 \pm 0.0$ ) were found to occur together, in 2 there was isolated ILC (ILC index - 1.06).

In all patients of group 2 statistically significant improvement of echoCG picture was also revealed during control follow-up period 12 months after complete myocardium revascularization (Table 5).

Complete normalization of systolic, diastolic and contractile functions of LV were observed in 6 individuals (in 4 patients from subgroup $A$, and in 2 from 
subgroup B). Abnormal basic functional myocardium parameters were revealed in 6 examined individuals: in 2 patients from subgroup A there was isolated ILC (ILC index - 1.06); in 1 patient in subgroup $B D D(E / A-$ 0.97 ) and ILC (ILC index - 1.18) occurred together; in 1 patient there was a combination of SD (EF - 49\%) and ILC (ILC index - 1.12), and in 2 patients isolated ILC (ILC index $-1.15 \pm 0.04$ ) was found.

The analysis of echoCG dynamics in patients with ACS in medium terms after the operative intervention convincingly showed, that 12 months after the operation patients with retroperfusion of the cardiac muscle (subgroups A) had statistically significantly better parameters of local contractility and diastolic function relative to the patients without supporting circulation (subgroups B) during percutaneous coronary intervention. Significant differences between the parameters of systolic function in subgroups $A$ and $B$ were found only in patients with NSTE-ACS (Table 6).

Discussion. The adequate selection of the quantity and quality of patients with ACS in the analyzed groups allowed us to conduct correct comparative investigation. All 24 examined patients, composing group 1 and 2, suffered from complex multivessel lesion of the coronary arteries. Evidence of symptom-associated hemodynamically significant damage in the zone of LCAT and ADA region promoted essential initial abnormalities of the basic functional parameters of LV myocardium [14]. A high probability of a long-running endovascular intervention, accompanied by a serious aggravation of LV anterior wall ischemia in the course of angioplasty and stenting, might result in a great risk of percutaneous coronary intervention and hospital lethality as a whole. It was such a "difficult" for endovascular correction cohort of ACS patients with STE-ACS and NSTE-ACS on electrocardiogram $[15,16]$, that encouraged us to form subgroups A, i.e. patients with retroperfusion support of myocardium.

12 months after complete myocardium revascularization, complete restoration of LV myocardium function was found to occur only in 6 patients of group 1 and 7 patients of group 2, in spite of the evident marked positive dynamics of echoCG picture in the examined patients with ACS. This situation is likely to be caused by ischemic heart disease in all 24 patients started long before the onset of ACS. MI in the history of 5 patients of group 1 and 6 patients in group 2, and concomitant pathology in the form of hypertension and diabetes mellitus in the majority of patients in both groups were serious reasons for forming ischemic remodeling of LV, and consequently, occurrence of SD, DD and ILC long before the first contact with a physician at the stage of acute coronary syndrome [17]. Acute aggravation of myocardium ischemia leads to a rapid worsening of the basic functional parameters of LV myocardium, and timely and maximally complete revascularization contributes to the slower restoration of energy and functional condition of the cardiac muscle (reverse remodeling). Of great importance is the fact, that reverse remodeling in patients with ACS may be completed during 6-12 months after myocardium reperfusion, whereas ischemic remodeling may take 1224 months since the onset of acute ischemic damage [18]. Therefore complete restoration of LV function may not be revealed in medium terms after the operation despite the positive dynamics of the echoCG picture. We observed the situation like that in the patients of both groups in our investigation.

At present it is known, that the time and completeness of myocardium function restoration in patients with ACS and multivessel damage of the coronary bloodstream after timely and complete revascularization of the myocardium is determined by the proportion of hibernating and stunned myocardium (viable myocardium), and also fibrous scar tissue (nonviable myocardium) in the acute ischemia region [19]. Sometimes the volume of nonviable myocardium is increased due to much more reduction of the initially decreased reserve of the coronary flow in ACS patients in the course of prolonged step-by-step angioplasty with stenting, accompanied by a long-term reduction of the antegrade blood flow [17]. Application of the local system supporting the coronary blood flow (retrograde myocardium perfusion) enables improvement of cardiac muscle blood supply mainly on the LV anterior wall and prevention of a negative effect of the restricted antegrade blood flow in the long process of intervention [20-22].

Thus, using our technique of endovascular retrograde cardiac blood supply, we observed significant differences between the parameters of diastolic function and segmentary contractility in subgroups A and B in ACS patients with STE-ACS and NSTE-ACS. Insignificant positive dynamics was found out only in patients with STE-ACS when analyzing systolic LV function in subgroups $A$ and $B 12$ months after the operation (EF in subgroup $A-59.3 \pm 4.8 \%$; in subgroup $B-56.2 \pm 5.1 \%$; $\mathrm{p}=0.240$ ).

Conclusion. Application of a novel technique of supporting retrograde myocardium blood supply, the principle of which is in the selective temporary arterializations of the large cardinal vein during a longterm endovascular correction of multivessel lesion of the coronary arterial circulation with localization of clinical picture-dependent damage in the zone of the left coronary artery trunk, anterior descending artery and large diagonal branches, which protects the myocardium from intraoperative aggravation of cardiac muscle acute ischemia, improves diastolic function and local myocardium contractility in patients 12 months after endovascular intervention.

Retrograde perfusion of the left ventricular wall may be considered a very useful procedure during complete endovascular revascularization in high-risk patients with acute coronary syndrome. 
Study Funding and Conflicts of Interest. This study was funded by the authors. There is no topic specific conflicts of interest related to the authors of this study.

\section{References}

1. Myat A., Patel N., Tehrani S., Banning A.P., Redwood S.R., Bhatt D.L. Percutaneous circulatory assist devices for high-risk coronary intervention. JACC Cardiovasc Interv 2015; 8(2): 229244, http://dx.doi.org/10.1016/j.jcin.2014.07.030.

2. Kolh P., Windecker S., Alfonso F., Collet J.P., Cremer J., Falk V., et al. 2014 ESC/EACTS Guidelines on myocardial revascularization: the Task Force on Myocardial Revascularization of the European Society of Cardiology (ESC) and the European Association for Cardio-Thoracic Surgery (EACTS). Developed with the special contribution of the European Association of Percutaneous Cardiovascular Interventions (EAPCI). Eur J Cardiothorac Surg 2014; 46(4): 517-592, http://dx.doi.org/10.1093/ejcts/ezu366.

3. O'Gara P.T., Kushner F.G., Ascheim D.D., Casey D.E. Jr., Chung M.K., de Lemos J.A., et al. 2013 ACCF/AHA guideline for the management of ST-elevation myocardial infarction: a report of the American College of Cardiology Foundation/American Heart Association Task Force on Practice Guidelines. J Am Coll Cardiol 2013; 61(4): e78-e140, http://dx.doi.org/10.1016/ j.jacc.2012.11.019.

4. Amsterdam E.A., Wenger N.K., Brindis R.G., Casey D.E. Jr., Ganiats T.G., Holmes D.R. Jr., et al. 2014 AHA/ ACC guideline for the management of patients with non-STelevation acute coronary syndromes: a report of the American College of Cardiology/American Heart Association Task Force on Practice Guidelines. Circulation 2014; 130(25): e344-e426, http://dx.doi.org/10.1161/CIR.0000000000000134.

5. Sjauw K.D., Engström A.E., Vis M.M., van der Schaaf R.J., Baan J. Jr., Koch K.T., de Winter R.J., Piek J.J., Tijssen J.G., Henriques J.P. A systematic review and metaanalysis of intra-aortic balloon pump therapy in ST-elevation myocardial infarction: should we change the guidelines? Eur Heart J 2009; 30(4): 459-468, http://dx.doi.org/10.1093/ eurheartj/ehn602.

6. Perera D., Stables R., Thomas M., Booth J., Pitt M., Blackman D., de Belder A., Redwood S.; BCIS-1 investigators. Elective intra-aortic balloon counterpulsation during high-risk percutaneous coronary intervention: a randomized controlled trial. JAMA 2010; 304(8): 867-874, http://dx.doi.org/10.1001/ jama.2010.1190.

7. Shah R., Thomson A., Atianzar K., Somma K., Mehra A., Clavijo L., Matthews R.V., Shavelle D.M. Percutaneous left ventricular support for high-risk $\mathrm{PCl}$ and cardiogenic shock: who gets what? Cardiovasc Revasc Med 2012; 13(2): 101-105, http://dx.doi.org/10.1016/j.carrev.2012.01.003.

8. Maini B., Naidu S.S., Mulukutla S., Kleiman N., Schreiber T., Wohns D., Dixon S., Rihal C., Dave R., O'Neill W. Real-world use of the Impella 2.5 circulatory support system in complex high-risk percutaneous coronary intervention: The USpella Registry. Catheter Cardiovasc Interv 2012; 80(5): 717725, http://dx.doi.org/10.1002/ccd.23403.

9. Froesch P., Martinelli M., Meier P., Cook S., Hullin R., Windecker S., Mohacsi P., Meier B. Clinical use of temporary percutaneous left ventricular assist devices. Catheter Cardiovasc
Interv 2011; 78(2): 304-313, http://dx.doi.org/10.1002/ ccd.22948.

10. Klein L.W., Krone R.J. Angiographic characterization of lesion morphology. Cardiac Interventions Today 2008; Jul/Aug: 44-49.

11. Belov Yu.V. Varaksin V.A. Postinfarktnoe remodelirovanie levogo zheludochka serdtsa. Ot kontseptsii k khirurgicheskomu lecheniyu [Post-infarction left ventricular remodeling. From concept to surgical treatment]. Moscow: DeNovo; 2002.

12. Shiller N.B., Osipov M.A. Klinicheskaya ekhokardiografiya [Clinical echocardiography]. Moscow: Praktika; 2005.

13. Petrov V.I., Nedogoda S.V. Meditsina, osnovannaya na dokazatel'stvakh [Evidence-based medicine]. Moscow: GeotarMedia; 2009.

14. Bandeali S.J., Stone S., Huang H.D., Kayani W.T., Wilson J.M., Birnbaum Y. Comparison of segmental wall motion abnormalities on echocardiography in patients with anteroseptal versus extensive anterior wall ST-segment elevation myocardial infarction. J Electrocardiol 2012; 45(6): 551-555, http://dx.doi. org/10.1016/j.jelectrocard.2012.07.001.

15. Stretch R., Sauer C.M., Yuh D.D., Bonde P. National trends in the utilization of short-term mechanical circulatory support: incidence, outcomes, and cost analysis. J Am Coll Cardiol 2014; 64(14): 1407-1415, http://dx.doi.org/10.1016/ j.jacc.2014.07.958.

16. Incorvati R.L., Tauberg S.G., Pecora M.J., Macherey R.S., Krucoff M.W., Dianzumba S.B., Donohue B.C. Clinical application of coronary sinus retroperfusion during high risk percutaneous transluminal coronary angioplasty. J Am Coll Cardiol 1993; 22(1): 127-134, http://dx.doi.org/10.1016/07351097(93)90826-m.

17. Zhang J., McDonald K.M. Bioenergetic consequences of left ventricular remodeling. Circulation 1995; 92(4): 1011-1019, http://dx.doi.org/10.1161/01.cir.92.4.1011.

18. Carluccio E., Biagioli P., Alunni G., Murrone A., Giombolini C., Ragni T., Marino P.N., Reboldi G., Ambrosio G. Patients with hibernating myocardium show altered left ventricular volumes and shape, which revert after revascularization. $J$ Am Coll Cardiol 2006; 47(5): 969-977, http://dx.doi.org/10.1016/ j.jacc.2005.09.064.

19. Perutskiy D.N., Makeeva T.I., Konstantinov S.L. The main concepts of post-infarction left ventricular remodeling. Nauchnye vedomosti BelGU. Meditsina. Farmatsiya 2011; 10: 51-59.

20. Kar S., Drury J.K., Hajduczki I., Eigler N., Wakida Y., Litvack F., Buchbinder N., Marcus H., Nordlander R., Corday E. Synchronized coronary venous retroperfusion for support and salvage of ischemic myocardium during elective and failed angioplasty. J Am Coll Cardiol 1991, 18(1): 271-282, http:II dx.doi.org/10.1016/s0735-1097(10)80249-9.

21. Costantini C., Sampaolesi A., Serra C.M., Pacheco G., Neuburger J., Conci E., Haendchen R.V. Coronary venous retroperfusion support during high risk coronary angioplasty in patients with unstable angina. preliminary experience. J Am Coll Cardiol 1991; 18(1): 283-292, http://dx.doi.org/10.1016/s07351097(10)80250-5.

22. Berland J., Farcot J.C., Barrier A, Dellac A., Gamra H., Letac B. Coronary venous synchronized retroperfusion during percutaneous transluminal coronary angioplasty of left anterior descending coronary artery. Circulation 1990; 81(4 Suppl): $35-42$. 